\title{
Brief eclectic psychotherapy $v$. eye movement desensitisation and reprocessing therapy for post-traumatic stress disorder: randomised controlled trial
}

Mirjam J. Nijdam, Berthold P. R. Gersons, Johannes B. Reitsma, Ad de Jongh and Miranda Olff

\section{Background}

Trauma-focused cognitive-behavioural therapy (CBT) and eye movement desensitisation and reprocessing therapy (EMDR) are efficacious treatments for post-traumatic stress disorder (PTSD), but few studies have directly compared them using well-powered designs and few have investigated response patterns.

\section{Aims}

To compare the efficacy and response pattern of a traumafocused CBT modality, brief eclectic psychotherapy for PTSD, with EMDR (trial registration: ISRCTN64872147)

\section{Method}

Out-patients with PTSD were randomly assigned to brief eclectic psychotherapy $(n=70)$ or EMDR $(n=70)$ and assessed at all sessions on self-reported PTSD (Impact of Event Scale - Revised). Other outcomes were clinician-rated PTSD, anxiety and depression.

\section{Results}

Both treatments were equally effective in reducing PTSD symptom severity, but the response pattern indicated that EMDR led to a significantly sharper decline in PTSD symptoms than brief eclectic psychotherapy, with similar drop-out rates (EMDR: $n=20$ (29\%), brief eclectic psychotherapy: $n=25(36 \%))$. Other outcome measures confirmed this pattern of results.

\section{Conclusions}

Although both treatments are effective, EMDR results in a faster recovery compared with the more gradual improvement with brief eclectic psychotherapy.

\section{Declaration of interest}

A.d.J. teaches and supervises clinical psychologists and psychiatrists in psychological trauma and its treatment by means of seminars, workshops and conferences, for which the participants pay a fee. He is also director and shareholder of a trauma treatment unit. For both activities he has the formal permission of the executive board of the University of Amsterdam to which he is affiliated.
After experiencing an event in which one's life is in imminent danger and one feels completely helpless, the conditional risk for developing post-traumatic stress disorder (PTSD) is $9-14 \% .^{1-3}$ Two trauma-focused psychotherapy methods, trauma-focused cognitive-behavioural therapy (CBT) and eye movement desensitisation and reprocessing therapy (EMDR), are the most efficacious psychological treatments for PTSD currently available. Their effect sizes have proven to be equally large, ${ }^{4-6}$ and there are some indications that EMDR leads to faster recovery. ${ }^{7}$ However, no studies have compared these treatments directly in sufficiently powered designs, and few studies have investigated the response pattern. The PTSD guideline of the National Institute for Health and Clinical Excellence (NICE) ${ }^{4}$ has also emphasised that adequately powered randomised trials should be conducted to compare these treatments, and to provide information about their value in clinical practice. Therefore, our aim was to conduct a well-powered randomised trial comparing the efficacy of, and response patterns to, treatment with EMDR and brief eclectic psychotherapy for patients with PTSD resulting from various types of psychological trauma. Brief eclectic psychotherapy was originally developed in The Netherlands and classified as a trauma-focused CBT in accordance with the NICE guideline. ${ }^{4}$ Although it includes some elements of other therapeutic schools, its main treatment components overlap with those of other trauma-focused CBT interventions. Some elements of 'practical trials' (trials that include elements of effectiveness designs) were included in the current trial to increase the extent to which results can be generalised to routine clinical practice and inform healthcare decisions. ${ }^{8}$ In line with a previous pilot study, ${ }^{7}$ we hypothesised that EMDR would lead to faster improvements in PTSD symptomatology than brief eclectic psychotherapy and that the improvements at the end would be equal. Trial registration: Dutch Trial Register, number NTR46 and ISRCTN64872147.

\section{Method}

Participants were recruited from the Centre for Psychological Trauma of the Academic Medical Centre in Amsterdam, The Netherlands, between December 2003 and January 2009 following ethics committee approval. Participants were civilian trauma survivors, who were referred to our centre by general practitioners, victim support workers, occupational physicians and other Academic Medical Centre departments. If a PTSD diagnosis was presumed at intake, individuals were approached for the study. After potential participants received a complete description of the study, written informed consent was obtained.

\section{Study entry criteria}

Study entry criteria were: a PTSD diagnosis according to DSM-IV; ${ }^{9}$ a single traumatic event (which had stopped at the time of inclusion) that led to the development of PTSD; age between 18 and 65 years; and mastery of the Dutch language. Exclusion criteria were: acute suicidality; current severe major depressive disorder or current severe alcohol or substance dependence according to DSM-IV (patients were allowed to enter after initial treatment of these 
disorders); lifetime psychotic disorder according to DSM-IV; and severe personality disorder according to the Structured Clinical Interview for DSM-IV Axis II Disorders (SCID-II) ${ }^{10}$ and DSM-IV. Patients with a history of earlier traumatic events were allowed to participate in the trial. Prior to entering the trial, patients who were on parallel pharmacological treatment were required to be on a stable medication dose for at least 4 weeks, and patients with prior alcohol or substance dependence were required to be abstinent for at least 3 months. During treatment, medication dosage was maintained as much as possible, but in agreement with clinical guidelines patients were allowed to stop anxiolytic medication to better engage in trauma processing. Participants were not allowed to attend any other trauma-focused intervention during their treatment in the trial.

\section{Design}

The study was a randomised controlled trial in which brief eclectic psychotherapy was compared with EMDR. The choice of brief eclectic psychotherapy, instead of another trauma-focused CBT intervention, was made because it explicitly combines the most effective ingredients of trauma-focused CBT such as psychoeducation, imaginal exposure, cognitive restructuring and writing assignments. Its effect sizes in previous studies with similar populations ${ }^{11,12}$ are equal to those of other trauma-focused cognitive-behavioural interventions. ${ }^{13}$

Participants were randomised to brief eclectic psychotherapy or EMDR in a parallel design. Random assignment was done on a 1:1 basis by a computer program, with a weighted maximum of subscribing four times the same treatment in a row. To ensure masking of assessors, one psychologist who had no other engagement in the study, had access to the computer program, kept a log file of all random assignments and assigned the patients to the therapists.

Both EMDR and brief eclectic psychotherapy were carried out according to treatment manuals and administered as in clinical practice, allowing for the number of sessions to vary depending on recovery. Leading trainers and supervisors in brief eclectic psychotherapy (B.P.R.G.) and EMDR (A.d.J.) were included in the study to control for an investigator allegiance effect. The primary outcome measure for self-reported PTSD symptoms was the Impact of Event Scale - Revised (IES-R), ${ }^{14}$ which was administered at baseline and at every weekly treatment session, as well as during a final assessment at week 17 when treatments had finished. Secondary outcomes were clinician-rated PTSD, depressive and general anxiety symptoms, which were also assessed at baseline and at week 17 (i.e. second post-assessment). Brief eclectic psychotherapy differs from EMDR in treatment duration and consists of two phases. Therefore, at mid-term an additional assessment was scheduled (first post-assessment) that was conducted after the EMDR treatment (mean 6.5 sessions) and after the first phase of brief eclectic psychotherapy (6 sessions; Fig. 1).

\section{Interventions}

The therapists were psychiatry residents or master's level clinical psychologists, who received a 3-day level-I training for EMDR and for brief eclectic psychotherapy. Prior experience with PTSD treatment was not required. A total of 38 therapists delivered the treatments; of these 7 delivered both. They received biweekly group supervision. All sessions were audiotaped. Treatment adherence protocols were developed to rate six brief eclectic psychotherapy sessions (session 1, 2, 4, 11, 13 and 15) and three EMDR sessions (first, second and second to last). Treatment adherence coding systems were based on a previous brief eclectic psychotherapy study ${ }^{11}$ and an EMDR Fidelity Scale ${ }^{15}$ adapted for use with the Dutch EMDR protocol.

\section{Eye movement desensitisation and reprocessing}

The weekly EMDR sessions lasted $90 \mathrm{~min}$ and were applied according to the Dutch treatment manual. ${ }^{16}$ During EMDR therapy, the most distressing images of the traumatic event are identified and processed consecutively. After the patient has focused on an image with the corresponding negative cognition, the most distressing emotion and the bodily location of the emotion, the patient is continuously asked to follow the therapist's finger making saccadic movements in alternation with the patient's own associations. Current distress is rated every 5-10 min, until the distress level is 0 or 1 , after which a more positive cognition is introduced in relation to the target image. This procedure is repeated for the other distressing images and treatment sessions are terminated when the trauma memory feels neutral. Auditory bilateral stimulation was used if problems with eye movements were encountered (such as if they induced headaches).

\section{Brief eclectic psychotherapy}

Brief eclectic psychotherapy was applied according to a detailed manual $^{17}$ and consisted of weekly sessions of $45-60 \mathrm{~min}$ as administered in previous studies. ${ }^{11,12,18}$ The treatment was initially developed and applied in The Netherlands and 400 therapists have been trained in this country in the last decade. Its main treatment components are also used in other traumafocused CBT, such as psychoeducation, imaginal exposure, writing assignments and cognitive restructuring. Two main phases can be clearly distinguished; from session 2 to 6 imaginal exposure takes place, whereas sessions $7-15$ are dedicated to cognitive restructuring. Session 1 consists of psychoeducation and session 16 of a farewell ritual. Some of the elements in the second phase can also be understood from other therapeutic perspectives, including grief therapy, directive therapy and a psychodynamic approach. These elements include taking mementos to the treatment session (objects that are linked to the traumatic event, such as the clothes that the person was wearing at that time, to stimulate the imaginal exposure), performing a farewell ritual that is intended to symbolically leave the trauma behind, and giving meaning to the traumatic event. The exposure in brief eclectic psychotherapy is very detailed, using sensory memories to stimulate reliving and focusing on experiencing grief. The aim is to relive the whole traumatic event in detail - in parts, over several sessions.

\section{Measures}

Assessments were conducted by trained, independent, masked assessors who were master's level clinical psychologists or master's level psychology students supervised by an experienced clinical psychologist. To ensure comparability among assessors, biweekly supervision took place. Before all post-assessments, patients were instructed to avoid mentioning details about the content or length of their treatment to ensure masking.

The primary outcome measure for PTSD symptom severity was the IES-R. ${ }^{14}$ Unlike the original revised version in which categories from zero to four are used, the Dutch IES-R rates the frequency of each item in the preceding week as zero (not at all), one (rarely), three (sometimes) and five (often). Clinical PTSD diagnoses were established by means of the Structured Interview for PTSD (SI-PTSD), ${ }^{19}$ which operationalises the DSM-IV criteria for PTSD. To assess comorbid psychiatric 


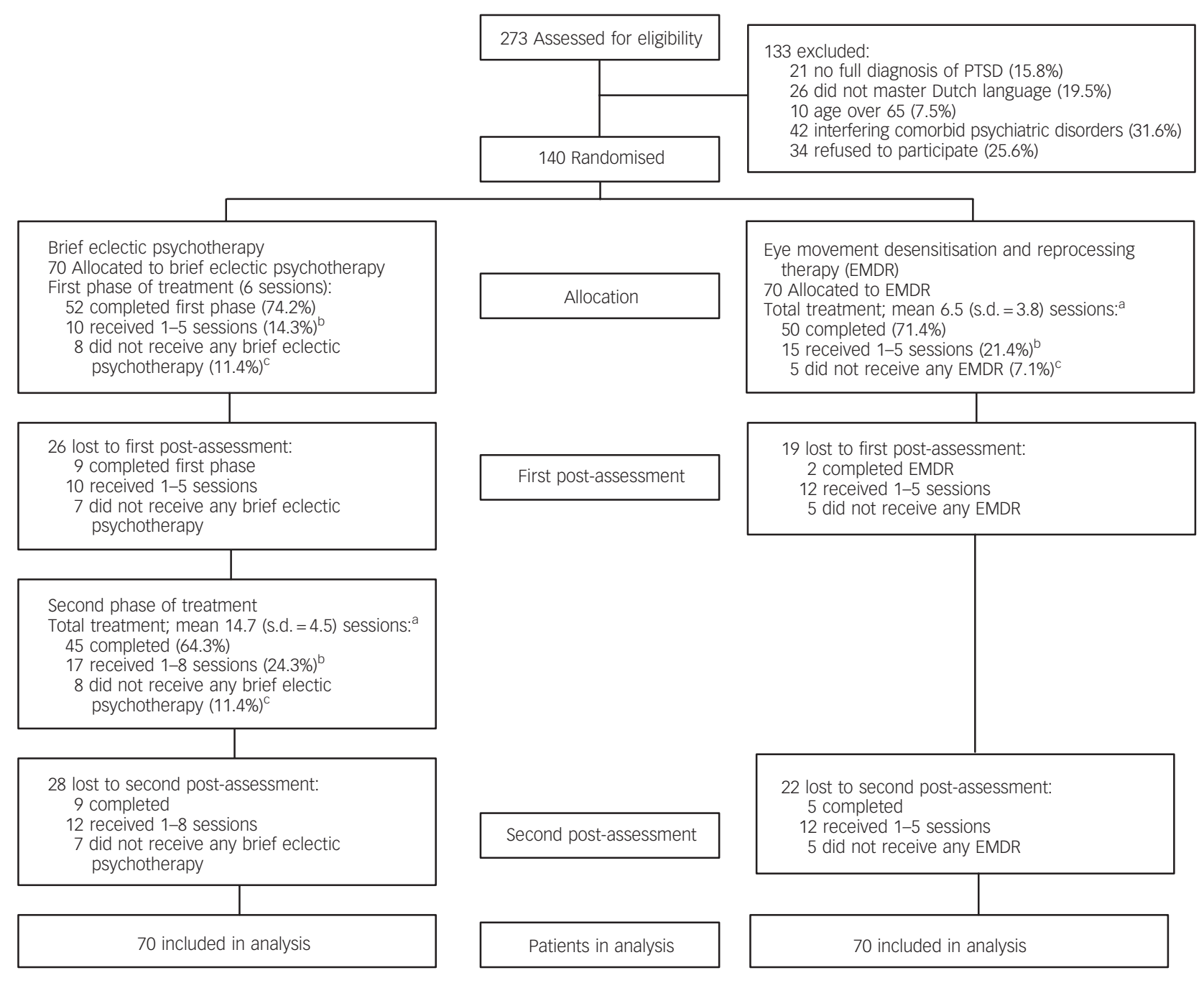

Fig. 1 Study design and patients' flow throughout trial.

PTSD, post-traumatic stress disorder. a. Number of sessions refers to the sessions completed by the participants. b. Patients who prematurely dropped out of treatment $(n=32)$ were contacted and the following reasons for drop-out were given: patient did not notice any improvement $(n=8)$, patient felt no further need to continue therapy ( $n=3)$, patient did not receive any treatment $(n=13)$ had health problems $(n=3)$ or withdrew from treatment before the first session $(n=10)$.

diagnoses, the Dutch version of the Structured Clinical Interview for DSM-IV Axis I Disorders (SCID-I) was administered. ${ }^{20,21}$ The Hospital Anxiety and Depression Scale (HADS) 22 was used to measure the severity of the general anxiety and depressive symptoms by self-report. All these measures have been widely used in trauma research and have been shown to have strong psychometric properties. ${ }^{23-26}$

\section{Data analysis}

Power calculations were based on post-treatment means and standard deviations of a previous study, ${ }^{12}$ which found a mean IES-R score of 49.5 (s.d. = 14.1) after brief eclectic psychotherapy for PTSD. Based on this previous study, we considered eight points on the IES-R a clinically relevant difference between the treatment conditions at post-assessment. For an equivalence design, a sample size of 47 patients per arm was needed (power $80 \%$ and two-sided significance level 0.05 ) to detect medium-sized treatment effects, taking into account a difference of less than eight points $(\delta)$ between the post-treatment means for equivalence of the two arms. Sample size per arm was set at 70 to allow for patient attrition of $30 \%$.
Chi-squared tests and independent $t$-tests were used to compare demographic and clinical characteristics between the treatment groups. Repeated measurement analyses were used to study changes over time between the treatment groups. We applied mixed linear models to take into account that measurements within the same individual are correlated, and to allow the model to calculate estimates when data were missing at certain assessments. An auto-regressive pattern was imposed on the covariance structure for measurements within the same individual (AR1). Mean scores for each outcome at the post-measurements (17 for IES-R and 2 for SI-PTSD and HADS) were modelled as a function of the intervention given (two levels), time since intervention (as a categorical variable with 17 or 2 levels), the baseline measurement (continuous), and the interaction between time and intervention. The main question, whether the response pattern was different between the treatment conditions, was evaluated by jointly testing whether the treatment difference was zero at all post-measurements. If the overall test was significant, we examined the size of the treatment effect by calculating the difference in mean scores between the treatment conditions at separate time points, with corresponding $95 \%$ confidence intervals 
in the linear mixed model. All analyses were carried out on an intent-to-treat basis, unless indicated otherwise. $P$-values of less than 0.05 were considered statistically significant and two-tailed tests were used throughout.

\section{Results}

\section{Participants}

Figure 1 shows the patient flow through the trial. Completer and drop-out rates did not significantly differ across treatment conditions $\left(\chi^{2}=1.08\right.$, d.f. $\left.=2, P=0.58\right)$. Table 1 displays the baseline and clinical characteristics of the two groups. No significant differences were found in clinical or demographic variables between the groups at baseline, except that the IES-R total score was significantly higher in the brief eclectic psychotherapy group than in the EMDR group (Table 1). Proportions of patients on psychoactive medication did not differ between treatment groups (Table 1). Over the 17 weeks of the trial, proportions of patients who changed psychoactive medication $(n=28)$ did not significantly differ between treatment conditions $\left(\chi^{2}=0.01\right.$, d.f. $\left.=1, P=0.91\right)$. Medication changes in most cases consisted of reduction or cessation of anxiolytics and these were also equally distributed across treatment conditions $\left(\chi^{2}=1.73\right.$, d.f. $\left.=1, P=0.18\right)$. Proportions of patients attending concurrent alternative treatments over the course of the trial did not significantly differ between treatment conditions $\left(\chi^{2}=1.54\right.$, d.f. $\left.=1, P=0.21\right)$. Alternative treatments consisted of physiotherapy $(n=17)$, alternative medicine $(n=15)$, supportive therapy $(n=10)$ and self-help groups $(n=1)$. Referrals at end of treatment $(n=18)$ were equally distributed across treatment conditions $\left(\chi^{2}=0.00\right.$, d.f. $\left.=1, P=1.00\right)$.

Reasons for treatment drop-out are given in Fig. 1. Participants who dropped out during sessions 4-8 scored higher on the IES-R than patients who continued treatment at those time points (all $P<0.001)$. Non-completers were significantly younger than treatment completers $(t=-3.60$, d.f. $=138, P<0.001)$ and more likely to be of non-Dutch origin $\left(\chi^{2}=11.76\right.$, d.f. $\left.=1, P<0.005\right)$.

\section{Treatment integrity}

Of all the interventions started, 48 participants $(37.8 \%, 24$ for each condition) were randomly selected for independent scoring of protocol adherence by two raters. The overall mean treatment integrity score was 78 for brief eclectic psychotherapy (s.d.=9.2) and 81 for EMDR (s.d.=14.7). Thus, according to the raters, on average $78-81 \%$ of the desired elements of the treatment protocol were applied during the interventions. Kappa values ranged between 0.54 for brief eclectic psychotherapy and 0.75 for EMDR, which can be considered indicative of adequate to good agreement between the raters. Treatment integrity scores did not significantly differ for brief eclectic psychotherapy and EMDR $(t=0.73$, d.f. $=37, P=0.48)$.

\section{Main outcomes}

Primary outcome

The results of the intent-to-treat analyses of the primary outcome IES-R are shown in Fig. 2. The mixed-model analysis demonstrated

Table 1 Demographic and clinical characteristics at baseline

\begin{tabular}{|c|c|c|c|c|c|c|}
\hline & \multirow{2}{*}{$\begin{array}{c}\text { Brief eclectic } \\
\text { psychotherapy group }(n=70)\end{array}$} & \multirow{2}{*}{$\begin{array}{l}\text { EMDR group } \\
\qquad(n=70)\end{array}$} & \multicolumn{4}{|c|}{ Analysis } \\
\hline & & & $\chi^{2}$ & $t$-test & d.f. & $P$ \\
\hline \multicolumn{7}{|l|}{ Characteristics } \\
\hline Age, years: mean (s.d.) & $37.3(10.6)$ & $38.3(12.2)$ & & 0.50 & 138 & 0.62 \\
\hline Female, $n(\%)$ & $43(61.4)$ & $36(51.4)$ & 1.42 & & 1 & 0.23 \\
\hline Education, $^{a} n(\%)$ & & & 2.35 & & 2 & 0.32 \\
\hline Low & $14(20.0)$ & $17(24.3)$ & & & & \\
\hline Middle & $38(54.0)$ & $29(41.4)$ & & & & \\
\hline High & $18(25.7)$ & $24(34.0)$ & & & & \\
\hline Ethnicity, $n$ (\%) & & & 0.76 & & 4 & 0.94 \\
\hline Dutch & $37(52.9)$ & $36(51.4)$ & & & & \\
\hline Surinamese & $10(14.3)$ & $8(11.4)$ & & & & \\
\hline Turkish & $7(10.0)$ & $6(8.6)$ & & & & \\
\hline Moroccan & $4(5.7)$ & $5(7.1)$ & & & & \\
\hline Other & $12(17.1)$ & $15(21.4)$ & & & & \\
\hline Trauma, ${ }^{\mathrm{b}} n(\%)$ & & & 1.46 & & & $0.94^{\mathrm{C}}$ \\
\hline Assault & $39(55.7)$ & $35(50.0)$ & & & & \\
\hline Sexual assault & $7(10.0)$ & $9(12.9)$ & & & & \\
\hline Accident & $12(17.1)$ & $14(20.0)$ & & & & \\
\hline Disaster & $4(5.7)$ & $6(8.6)$ & & & & \\
\hline War-related & $4(5.7)$ & $3(4.3)$ & & & & \\
\hline Other & $4(5.7)$ & $3(4.3)$ & & & & \\
\hline \multicolumn{7}{|l|}{ Clinical features } \\
\hline Earlier traumatic experiences, $n$ (\%) & $40(57.1)$ & $36(51.4)$ & 0.60 & & 1 & 0.44 \\
\hline Complex trauma & $15(21.4)$ & $11(15.7)$ & 0.76 & & 1 & 0.39 \\
\hline On psychoactive medication, $n$ (\%) & $30(42.9)$ & $29(41.4)$ & 0.03 & & 1 & 0.86 \\
\hline Antidepressants & $13(18.6)$ & $12(17.1)$ & 0.05 & & 1 & 0.83 \\
\hline Anxiolytics or opiates & $18(25.7)$ & $23(32.8)$ & 0.86 & & 1 & 0.35 \\
\hline Propranolol & $3(4.3)$ & $2(2.9)$ & & & & 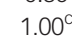 \\
\hline Antipsychotics & $1(1.4)$ & $1(1.4)$ & & & & $1.00^{\mathrm{C}}$ \\
\hline Time since trauma in months, mean (s.d.) & $31.5(50.6)$ & $29.1(62.0)$ & & 0.25 & 138 & 0.80 \\
\hline IES-R PTSD total, mean (s.d.) & $79.9(16.9)$ & $72.8(20.7)$ & & 2.25 & 137 & $0.03^{*}$ \\
\hline \multicolumn{7}{|c|}{$\begin{array}{l}\text { EMDR, eye movement desensitisation and reprocessing; IES-R, Impact of Event Scale - Revised; PTSD, post-traumatic stress disorder. } \\
\text { a. Low: completed elementary school or lower vocational education. Middle: completed high-school or middle-level vocational education. High: completed pre-university, college } \\
\text { or university degree. } \\
\text { b. All met criterion A1 and A2 of DSM-IV. } \\
\text { c. Fisher's exact test. } \\
\text { *Significant at } 0.05 \text { level. }\end{array}$} \\
\hline
\end{tabular}


a significant main effect of time $(F=17.99$, d.f. $=1065, P<0.001)$, a significant main effect of treatment condition $(F=12.20$, d.f. $=169, P<0.005)$ and a significant interaction between time and treatment condition $(F=4.00$, d.f. $=1065, P<0.001)$. The response pattern proved to be significantly different for brief eclectic psychotherapy and $\operatorname{EMDR}(t=3.49$, d.f. $=169$, $P<0.005)$. The mean estimated difference on the IES-R between the treatment conditions across the 17 measurements was 13.10 (95\% CI 5.69-20.50), corresponding with a large effect size (Cohen's $d=0.75$ ). Mean IES-R total scores at the second postassessment for brief eclectic psychotherapy were 38.0 (s.d. $=34.4$, $n=41$ ) and for EMDR 28.5 (s.d. $=29.6, n=48$ ). At the second post-assessment, the difference between the treatment conditions was no longer significant $(t=0.70$, d.f. $=340, P=0.48$; mean estimated difference 3.70 ( $95 \%$ CI -6.63 to 14.03 )).

Additional analyses comparing IES-R scores after every two EMDR sessions with every three brief eclectic psychotherapy sessions showed that the response pattern was also significantly different for brief eclectic psychotherapy and EMDR $(t=2.12$, d.f. $=139, P<0.05)$. Improvement effect sizes from baseline to second post-assessment were large for both treatment conditions (Cohen's $d=1.55$ for brief eclectic psychotherapy and Cohen's $d=1.73$ for EMDR). The completers-only analysis yielded comparable results for the IES-R scores.

\section{Secondary outcomes}

The mixed-model analysis of the SI-PTSD showed a significant main effect of time $(F=37.06$, d.f. $=86, P<0.001)$, a significant main effect of treatment condition $(F=11.05$, d.f. $=98$, $P<0.005)$ and a significant interaction between time and treatment condition $(F=14.99$, d.f. $=86, P<0.001)$. The response pattern proved to be significantly different for brief eclectic psychotherapy and EMDR $(t=3.32$, d.f. $=98, P<0.005)$. Analysis by time point revealed that SI-PTSD scores were significantly lower for EMDR than for brief eclectic psychotherapy at the first post-assessment, but at the second post-assessment the difference was no longer significant (Table 2). Improvement effect sizes from baseline to second post-assessment were large for both treatment conditions (Cohen's $d=1.95$ for brief eclectic psychotherapy and Cohen's $d=2.43$ for EMDR).

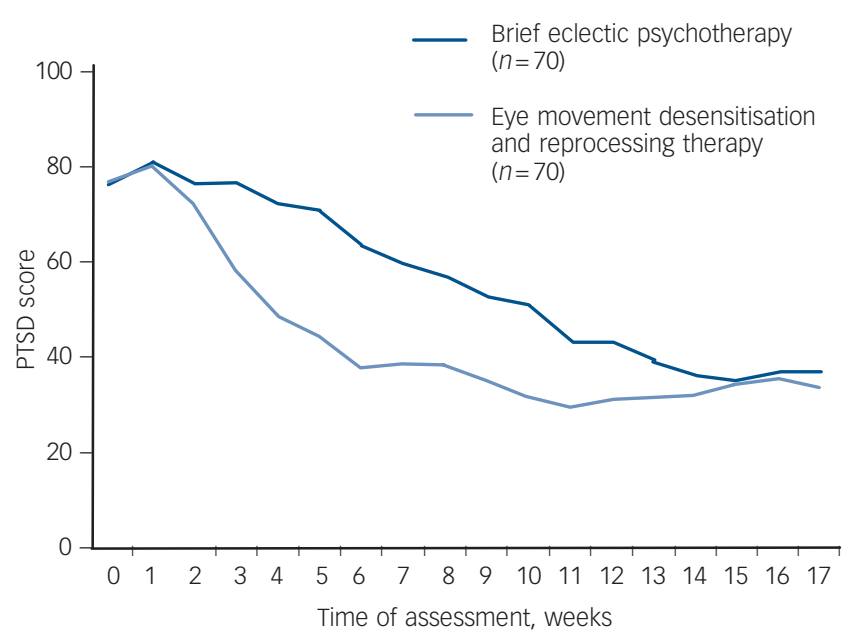

Fig. 2 Changes in post-traumatic stress disorder (PTSD) scores on the Impact of Event Scale - Revised for intent-to-treat analysis.

Mean values at assessment points from a repeated measures model adjusted for baseline value of PTSD score.

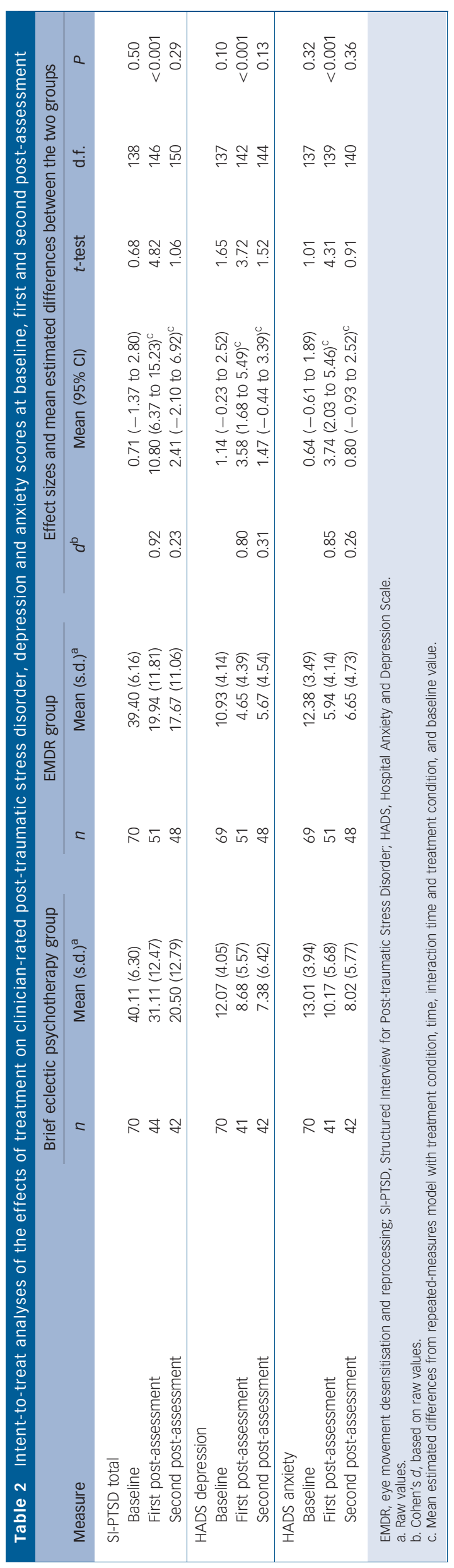


The mixed-model analysis on HADS depression scores revealed a significant main effect of treatment condition $(F=8.72$, d.f. $=97, \quad P<0.005)$ and a significant interaction between time and treatment condition $(F=5.61$, d.f. $=83$, $P<0.05)$, but no significant main effect of time $(P=0.63)$. The response pattern was significantly different for brief eclectic psychotherapy and EMDR $(t=2.95$, d.f. $=97, P<0.005)$. Analysis by time point showed that HADS depression scores were significantly lower for EMDR than for brief eclectic psychotherapy at the first post-assessment, but at the second post-assessment the difference was not significant anymore (Table 2). Improvement effect sizes from baseline to second post-assessment were large for both treatment conditions (Cohen's $d=0.87$ for brief eclectic psychotherapy and Cohen's $d=1.21$ for EMDR).

Mixed-model analysis on HADS anxiety scores revealed a significant main effect of time $(F=6.49$, d.f. $=83, P<0.05)$, a significant main effect of treatment condition $(F=8.43$, d.f. $=98$, $P<0.01)$ and a significant interaction between time and treatment condition $(F=14.90$, d.f. $=83, P<0.001)$. The response pattern was significantly different for brief eclectic psychotherapy and EMDR $(t=2.90$, d.f. $=98, P<0.01)$. Analysis by time point showed that HADS anxiety scores were significantly lower for EMDR than for brief eclectic psychotherapy at the first post-assessment, but at the second post-assessment the difference was no longer significant (Table 2). Improvement effect sizes from baseline to second post-assessment were large for both treatment conditions (Cohen's $d=1.01$ for brief eclectic psychotherapy and Cohen's $d=1.38$ for EMDR).

We found similar results in the completers-only analyses of the SI-PTSD, HADS depression and HADS anxiety scores.

Numbers of patients with psychiatric diagnoses at the first and second post-assessment are shown in Table 3. Significant differences were found for PTSD and major depressive disorder at the first post-assessment; other differences were not significant.

\section{Discussion}

The current randomised controlled trial showed that EMDR and brief eclectic psychotherapy had equal effects in terms of reduction of self-reported and clinician-rated PTSD symptoms, depressive symptoms and general anxiety symptoms when statistically controlling for pre-treatment differences. Across all outcomes, the response pattern was significantly different for EMDR and brief eclectic psychotherapy when accounting for baseline differences, indicating that EMDR led to faster symptom decline and brief eclectic psychotherapy to more gradual improvement.

\section{Efficacy and mechanism}

Several explanations can be found for the faster symptom reduction in EMDR. First, the type of exposure to the traumatic event is different for brief eclectic psychotherapy and EMDR. In EMDR, short interrupted exposures to the 'hotspots' of the trauma are alternated with free association (which can involve moving quickly through scenes and associating with memories of other events that the person has experienced), and this associative process is seen as effective memory processing. ${ }^{27}$ During brief eclectic psychotherapy, on the other hand, in every exposure session a part of the trauma is relived in great detail with a focus on experiencing grief, leading to more gradual trauma processing. The differences in exposure type and duration may explain the initial gradual change observed in brief eclectic psychotherapy and the faster change in EMDR. Due to the repetitive nature of interventions in both traditional prolonged exposure and EMDR, it is possible that the effects would be obtained faster than in brief eclectic psychotherapy. Further symptom reduction was observed for brief eclectic psychotherapy during the second treatment phase. So in this treatment the cognitive restructuring part, in which patients reflect on their trauma story and its meaning for their life, proved to be important for recovery from PTSD. Possibly, adding this phase to EMDR, as is sometimes done in clinical practice, could lead to further symptom reduction as well. Further research could address this by detailed study of the effective 'ingredients' of these treatments.

A second possible explanation for faster improvement with EMDR is that the session duration is $30-45$ min longer than for brief eclectic psychotherapy. Additional analyses corrected for the difference in session duration still showed that EMDR led to faster symptom improvement. In this context it is interesting to note that strictly speaking the exposure time to the traumatic memory is shorter in EMDR than in brief eclectic psychotherapy. In brief eclectic psychotherapy the exposure is uninterrupted for 15-20 min, whereas in EMDR the exposure is limited to a few minutes in which the patient focuses on traumatic images. The session durations were those standard in clinical care and were therefore implemented as such in our study. Optimal session duration and frequency should be investigated in future research.

\begin{tabular}{|c|c|c|c|c|c|}
\hline & \multirow{2}{*}{$\begin{array}{l}\text { Brief eclectic psychotherapy group } \\
\qquad n / N(\%)\end{array}$} & \multirow{2}{*}{$\begin{array}{l}\text { EMDR group } \\
\qquad n / N(\%)\end{array}$} & \multicolumn{3}{|c|}{ Analysis } \\
\hline & & & $\chi^{2}$ & d.f. & $P$ \\
\hline \multicolumn{6}{|l|}{$\mathrm{PTSD}^{\mathrm{a}}$} \\
\hline Baseline & 70/70 (100) & 70/70 (100) & \multirow{3}{*}{19.38} & \multirow{3}{*}{1} & \\
\hline First post-assessment & 21/44 (47.7) & 4/51 (7.8) & & & $<0.001$ \\
\hline Second post-assessment & 6/42 (14.3) & $3 / 48(6.3)$ & & & $0.30^{\mathrm{b}}$ \\
\hline \multicolumn{6}{|l|}{ Major depressive disorder ${ }^{C}$} \\
\hline Baseline & $47 / 70(67.1)$ & $37 / 70(52.9)$ & 2.98 & 1 & 0.08 \\
\hline First post-assessment & $16 / 44(36.4)$ & $7 / 51(13.7)$ & 6.60 & 1 & $<0.05$ \\
\hline Second post-assessment & 8/42 (19.0) & $7 / 48(14.6)$ & 0.32 & 1 & 0.57 \\
\hline \multicolumn{6}{|c|}{ Anxiety disorder other than PTSD ${ }^{c}$} \\
\hline Baseline & 14/70 (20.0) & 8/70 (11.4) & 1.94 & 1 & 0.16 \\
\hline First post-assessment & $4 / 44(9.1)$ & $5 / 51(9.8)$ & 0.01 & 1 & 0.91 \\
\hline Second post-assessment & $5 / 42(11.9)$ & $5 / 48(10.4)$ & 0.05 & 1 & 0.82 \\
\hline \multicolumn{6}{|c|}{$\begin{array}{l}\text { EMDR, eye movement desensitisation and reprocessing; PTSD, post-traumatic stress disorder. } \\
\text { a. Structured Interview for Post-traumatic Stress Disorder. } \\
\text { b. Fisher's exact test. } \\
\text { c. Structured Clinical Interview for DSM-IV Axis I Disorders. }\end{array}$} \\
\hline
\end{tabular}




\section{Comparison of our findings with previous studies}

The results of our study are in line with previous trials that compared other trauma-focused CBT approaches with EMDR (i.e. the pilot study that found that EMDR leads to faster reduction in PTSD symptoms than trauma-focused CBT after a few sessions ${ }^{7}$ and trials reporting that both treatments led to equal improvements on PTSD symptoms post-treatment ${ }^{7,28-32}$ ). Although the effects at the end-point were equal, it should be noted that the current study cannot address with certainty whether the treatments are equivalent at that point because the confidence interval of the primary outcome contains the pre-specified difference of eight points. ${ }^{33}$ This is attributable to the fact that the variance at the second post-assessment proved to be larger than we expected in our power analysis. The number of participants who completed their treatment and completed their assessment closely approximated the number of participants needed per arm in the power analysis, so we anticipate that drop out did not bias our results.

The magnitude of change in our trial is larger than in some previous studies. Although both baseline and post-treatment levels of the IES-R in our sample seem higher than in other studies, this is attributable to the different scoring method adopted by the Dutch version of the instrument. The PTSD improvement effect sizes from pre- to post-treatment for both brief eclectic psychotherapy and EMDR in our study were higher than those found across all active PTSD treatments in meta-analyses. ${ }^{13,34}$ The improvement effect sizes of EMDR in the current study were higher than the overall effect size for EMDR as reported in these meta-analyses, and brief eclectic psychotherapy improvement effect sizes were comparable with those of trauma-focused CBT.

\section{Strengths and limitations}

A strength of the current study is that we succeeded in comparing response patterns in two trauma-focused treatment protocols in a large, culturally diverse urban sample using a design that took into account sound methodology as well as clinical relevance. We emphasised scientific rigour by randomisation, protocol adherence, treatment integrity checks and independent assessment of outcome. The clinical meaningfulness of the results was increased by the inclusion of some elements of practical trials (trials that include elements of effectiveness designs), such as the inclusion of a heterogeneous trauma population, treatment duration dependent on the patients' recovery and the use of non-expert therapists.

We also recognise the limitations of our study. The foremost limitation is the drop out from both therapy and the assessments. However, the treatment drop-out rates of the current study are comparable with those in other trials. ${ }^{35-37}$ Interestingly, around $10 \%$ of patients dropped out before treatment, which might indicate a difficulty in starting trauma therapy. The drop-out rates from assessments were somewhat higher than in other studies, especially for brief eclectic psychotherapy. Nevertheless, for the primary outcome we were able to analyse a large proportion of the randomised patients because the data from the treatment sessions enabled us to calculate estimates of the response patterns if patients dropped out of later assessments.

A further limitation was that the number of brief eclectic psychotherapy exposure sessions fluctuated to a small degree, which could have exerted a small influence on our first post-assessment comparison. Finally, concurrent treatments such as pharmacological treatment, changes in pharmacological treatment and non-trauma-focused therapies may have contributed to the therapy effects for a minority of the patients. However, concurrent treatments were equally distributed across both brief eclectic psychotherapy and EMDR conditions and it may be presumed that they had an equal effect in both groups.

\section{Clinical implications}

In conclusion, this study demonstrates that both brief eclectic psychotherapy and EMDR are effective psychotherapeutic treatments, but EMDR may be a more time-efficient method for treating PTSD. Effect sizes and rates of diagnostic change were large for both treatment methods, indicating that the majority of people with PTSD benefit from trauma-focused psychotherapy. Symptom improvement appeared to occur at an earlier stage in EMDR than in brief eclectic psychotherapy, which may make patients more keen to choose this treatment method. However, brief eclectic psychotherapy may be preferred if patients value reflection on the trauma story and its meaning for their lives. Patient and therapist preferences have been shown to play an important role in the choice of treatment method. ${ }^{38}$ Because with both treatments patients dropped out, we should keep searching for new therapeutic strategies. ${ }^{39}$ Future studies also need to prioritise investigating the reasons for premature treatment drop-out. Possibly, more attention should be given to psychoeducation and motivation to overcome persistent avoidance. This may especially be true for younger patients, those from minority ethnic groups and those who do not show symptom improvement over the first sessions and who drop out from treatment more frequently. Furthermore, future research needs to determine which patient groups benefit most from which form of psychotherapy. Finally, investigating long-term treatment effects is essential in order to offer individuals the best possible treatment option.

\section{Funding}

This study was supported by the Academic Medical Centre, Amsterdam, The Netherlands.

\section{Acknowledgements}

The authors thank the patients who gave their consent to participate in this study. We also thank the therapists and assessors for their contribution to the study, and especially the coordinators Noor de Bruijn, Ineke Vrijlandt and Renée Hutter.

Mirjam J. Nijdam, MSc, Centre for Psychological Trauma, Department of Psychiatry, Academic Medical Centre at the University of Amsterdam, Amsterdam, The Netherlands; Berthold P. R. Gersons, MD, PhD, Centre for Psychological Trauma, Department of Psychiatry, Academic Medical Centre at the University of Amsterdam and Arq Psychotrauma Expert Group, Diemen, The Netherlands; Johannes B.

Reitsma, MD, PhD, Department of Clinical Epidemiology, Biostatistics \&

Reitsma, MD, PhD, Department of Clinical Epidemiology, Biostatistics \&
Bioinformatics, Academic Medical Centre of the University of Amsterdam,

Amsterdam, The Netherlands; Ad de Jongh, PhD, Department of Behavioral

Sciences, Academic Centre for Dentistry Amsterdam, University of Amsterdam and Vrije University, Amsterdam, The Netherlands, and School of Health Sciences, Salford University, Manchester, UK; Miranda Olff, PhD, Centre for Psychological Trauma, Department of Psychiatry, Academic Medical Centre at the University of Amsterdam and Arq Psychotrauma Expert Group, Diemen, The Netherlands

Correspondence: Mirjam J. Nijdam, MSC, Centre for Psychological Trauma, Department of Psychiatry, Academic Medical Centre at the University of Amsterdam, Meibergdreef 5, 1105 AZ Amsterdam, The Netherlands. Email:m.j.nijdam@amc.uva.nl

First received 8 Jul 2011, final revision 7 Oct 2011, accepted 19 Dec 2011

\section{References}

1 Breslau N, Kessler RC, Chilcoat HD, Schultz LR, Davis GC, Andreski P. Trauma and posttraumatic stress disorder in the community: the 1996 Detroit area survey of trauma. Arch Gen Psychiatry 1998; 55: 626-32.

2 Kessler RC, Sonnega A, Bromet E, Hughes M, Nelson CB. Posttraumatic stress disorder in the national comorbidity survey. Arch Gen Psychiatry 1995; 52: $1048-60$. 
3 De Vries GJ, Olff M. The lifetime prevalence of traumatic events and posttraumatic stress disorder in the Netherlands. J Trauma Stress 2009; 22 259-67.

4 National Collaborating Centre for Mental Health. Post-Traumatic Stress Disorder (PTSD): The Management of PTSD in Adults and Children in Primary and Secondary Care (CG 26). National Institute for Health and Clinical Excellence, 2005.

5 Foa EB, Keane TM, Friedman MJ, Cohen JA. Effective Treatments for PTSD: Practice Guidelines from the International Society for Traumatic Stress Studies (2nd edn). Guilford Press, 2008.

6 Seidler GH, Wagner FE. Comparing the efficacy of EMDR and trauma-focused cognitive-behavioural therapy in the treatment of PTSD: a meta-analytic study. Psychol Med 2006; 36: 1515-22.

7 Ironson G, Freund B, Strauss JL, Williams J. Comparison of two treatments for traumatic stress: a community-based study of EMDR and prolonged exposure. J Clin Psychol 2002; 58: 113-28.

8 Schnurr PP. The rocks and hard places in psychotherapy outcome research. J Trauma Stress 2007; 20: 779-92.

9 American Psychiatric Association. Diagnostic and Statistical Manual of Mental Disorders (4th edn) (DSM-IV). APA, 1994.

10 First MB, Gibbon M, Spitzer RL, Williams JBW, Benjamin LS. Structured Clinical Interview for DSM-IV Axis II Personality Disorders Self-Report. American Psychiatric Association, 1997

11 Lindauer RJL, Gersons BPR, Van Meijel EPM, Blom K, Carlier IVE, Vrijlandt I, et al. Effects of brief eclectic psychotherapy in outpatients with posttraumatic stress disorder: randomized clinical trial. J Trauma Stress 2005; 18: 205-12.

12 Olff M, De Vries GJ, Güzelcan Y, Assies J, Gersons BPR. Changes in cortiso and DHEA plasma levels after psychotherapy for PTSD. Psychoneuroendocrinology 2007; 32: 619-26.

13 Bradley R, Greene J, Russ E, Dutra L, Westen D. A multidimensional metaanalysis of psychotherapy for PTSD. Am J Psychiatry 2005; 162: 214-27.

14 Weiss DS, Marmar CR. The impact of event scale - revised. In Assessing Psychological Trauma and PTSD: A Handbook for Practitioners (eds JP Wilson RM Keane): 399-411. Guilford Press, 1997.

15 Korn DL, Zangwill W, Lipke H, Smyth NJ. EMDR Fidelity Scale. EMDRIA Research Committee, 2001

16 De Jongh A, Ten Broeke E. Handboek EMDR: Een Geprotocolleerde Behandelmethode voor de Gevolgen van Psychotrauma [Handbook EMDR: A Manualized Treatment Method for the Consequences of Psychotrauma]. Harcourt Assessment, 2004.

17 Gersons BPR, Carlier IVE, Olff M. Protocol Brief Eclectic Psychotherapy for Posttraumatic Stress Disorder. Academic Medical Center, University of Amsterdam, 2004

18 Gersons BPR, Carlier IVE, Lamberts RD, van der Kolk BA. Randomized clinica trial of brief eclectic psychotherapy for police officers with posttraumatic stress disorder. J Trauma Stress 2000; 13: 333-47.

19 Davidson JR, Malik MA, Travers J. Structured interview for PTSD (SIP): psychometric validation for DSM-IV criteria. Depress Anxiety 1997; 5: 127-9.

20 Spitzer B, Gibbon RL, Janet M, Janet W. Structured Clinical Interview for DSM-IV Axis I Disorders - Patient Edition (SCID I/P, version 2.0). American Psychiatric Press, 1996.

21 Van Groenestein MAC, Akkerhuis GW, Kupka RW, Schneider N, Nolen WA. Gestructureerd Klinisch Interview voor de Vaststelling van DSM-IV As I Stoornissen [Structured Clinical Interview for the Diagnosis of DSM-IV Axis I Disorders]. Swets \& Zeitlinger BV, 1999.
22 Zigmond AS, Snaith RP. The Hospital Anxiety and Depression Scale. Acta Psychiatr Scand 1983; 67: 361-70.

23 Creamer M, Bell R, Failla S. Psychometric properties of the Impact of Event Scale - Revised. Behav Res Ther 2003; 41: 1489-96.

24 Carlier IV, Lamberts RD, Van Uchelen AJ, Gersons BP. Clinical utility of a brief diagnostic test for posttraumatic stress disorder. Psychosom Med 1998; 60: 42-7

25 Zanarini MC, Frankenburg FR. Attainment and maintenance of reliability of Axis I and II disorders over the course of a longitudinal study. Compr Psychiatry 2001; 42: 369-74.

26 Spinhoven P, Ormel J, Sloekers PP, Kempen GI, Speckens AE, Van Hemert AM. A validation study of the Hospital Anxiety and Depression Scale (HADS) in different groups of Dutch subjects. Psychol Med 1997; 27: 363-70.

27 Schubert S, Lee CW. Adult PTSD and its treatment with EMDR: a review of controversies, evidence, and theoretical knowledge. J EMDR Pract Res 2009; 3: $117-32$.

28 Vaughan K, Armstrong MS, Gold R, O'Connor N, Jenneke W. A trial of eye movement desensitization compared to image habituation training and applied muscle relaxation in posttraumatic stress disorder. J Behav Ther Exp Psychiatry 1994; 25: 283-91.

29 Lee C, Gavriel H, Drummond P, Richards J, Greenwald R. Treatment of PTSD: stress inoculation training with prolonged exposure compared to EMDR. J Clin Psychol 2002; 58: 1071-89.

30 Power K, McGoldrick T, Brown K, Buchanan R, Sharp D, Swanson V, et al A controlled comparison of eye movement desensitization and reprocessing versus exposure plus cognitive restructuring versus waiting list in the treatment of post-traumatic stress disorder. Clin Psychol Psychother 2002; 9: $299-318$

31 Taylor S, Thordarson DS, Maxfield L, Fedoroff IC, Lovell K, Ogrodniczuk J. Comparative efficacy, speed and adverse effects of three PTSD treatments: exposure therapy, EMDR, and relaxation training. J Consult Clin Psychol 2003; 71: $330-8$.

32 Rothbaum BO, Astin MC, Marsteller F. Prolonged exposure versus eye movement desensitization and reprocessing (EMDR) for PTSD rape victims. J Trauma Stress 2005; 18: 607-16.

33 Jones $B$, Jarvis $P$, Lewis JA, Ebbutt AF. Trials to assess equivalence: the importance of rigorous methods. BMJ 1996; 313: 36-9.

34 Bisson Jl, Ehlers A, Matthews R, Pilling S, Richards D, Turner S. Psychological treatments for chronic post-traumatic stress disorders. Systematic review and meta-analysis. Br J Psychiatry 2007; 190: 97-104.

35 Hembree EA, Foa EB, Dorfan NM, Street GP, Kowalski J, Tu X. Do patients drop out prematurely from exposure therapy for PTSD? J Trauma Stress 2003; 16: 555-62.

36 Schnurr PP, Friedman MJ, Engel CC, Foa EB, Shea MT, Chow BK, et al. Cognitive behavioural therapy for posttraumatic stress disorder in women: a randomized controlled trial. JAMA 2007; 297: 820-30.

37 Schottenbauer MA, Glass CR, Arnkoff DB, Tendick V, Gray SH. Nonresponse and dropout rates in outcome studies on PTSD: review and methodological considerations. Psychiatry 2008; 71: 134-68.

38 Van Minnen A, Hendriks L, Olff M. When do trauma experts choose exposure therapy for PTSD patients? A controlled study of therapist and patient factors. Behav Res Ther 2010; 48: 312-20.

39 Schnyder U. Why new psychotherapies for posttraumatic stress disorder? Psychother Psychosom 2005; 74: 199-201. 